\title{
Preparation and characterization of doxorubicin-containing liposomes. II. Loading capacity, long-term stability and doxorubicin-bilayer interaction mechanism
}

\author{
D.J.A. Crommelin * and L. van Bloois \\ Dept. of Pharmaceutics, Subfaculty of Pharmacy, Cniversity of Utrecht, Catharijnesingel 60. \\ 3511 GH Utrecht (The Netherlands)
}

(Received April 11th, 1983)

(Accepted May 12th, 1983)

\begin{abstract}
Summary
Doxorubicin loading capacity was determined for negative (phosphatidylcholine-cholesterol-phosphatidylserine, 10:4:1) and positive (phosphatidylcholine-cholesterol-stearylamine $10: 4: 3$ ) liposomes prepared according to the "film" method with doxorubicin added to the phospholipids before film formation. Doxorubicin association depended on the initial ratio of doxorubicin/phospholipid, the charge and the size. The maximum loading capacity was $60-75 \mathrm{mmol}$ doxorubicin/mol phospholipid for the negative and about $55 \mathrm{mmol}$ doxorubicin/mol phospholipid for positive, non-filtered, multilamellar liposomes. Filtration or ultrasonication/ultracentrifugation reduced the doxorubicin-bilayer interaction. Loss on storage of doxorubicin from the filtered, multilamellar, or unilamellar vesicles decreased in the following order: filtered negative $<$ unilamellar positive < unilamellar negative vesicles. Positive unilamellar vesicles increased in particle size on storage. Negative liposomes were stable. $\zeta$-Potentials of positive or negative liposomes did not depend on the presence of associated doxorubicin. This makes a purely electrostatic mechanism of interaction between the drug and the bilayer unlikely. If doxorubicin was added to the phospholipid film in the hydration buffer a dramatic drop in doxorubicin-bilayer association was observed for positive liposomes. For negative liposomes the loading efficiency was unaffected.
\end{abstract}

* To whom correspondence should be addressed. 


\section{Introduction}

Intravenous injection of doxorubicin-containing liposomes has been reported to increase the therapeutic index of the drug in mice and rats (Rahman et al., 1980, Forssen and Tökes, 1981, Olson et al., 1982, Gabizon et al., 1982). For proper evaluation of the in vivo results a physicochemical characterization of the used liposomes is an essential prerequisite. In a previous article (Crommelin et al., 1983) we reported on the influence of the $\mathrm{pH}$ of the hydration buffer on size and loading capacity of positively and negatively charged liposomes containing doxorubicin. In this article we investigated: (1) the loading capacity as a function of the doxorubicin/phospholipid (PL) ratio; and (2) the release of the associated doxorubicin from the liposomes and particle size stability on storage. Besides, we tried to elucidate the mechanism of doxorubicin association with the bilayer. The lipid composition of both the positive and negative liposomes was kept constant throughout this study and corresponded to those used by Rahman et al. (1980) and Crommelin et al. (1983).

\section{Methods and Materials}

\section{Liposome preparation}

Liposome composition, chemicals used and preparation of positively and negatively charged multilamellar vesicles (MLV) were described earlier (Crommelin et al., 1983). The hydration medium ( $\mathrm{pH} \mathrm{4.0)}$ ) contained $0.01 \mathrm{M}$ Tris- $\mathrm{HCl}$ and $0.8 \%$ sodium chloride. Sizing of the liposomes was done either by sequential filtration throligh 600 and $200 \mathrm{~nm}$ membrane filters (Uni-pore polycarbonate, Biorad Lab.) (negative liposomes) or by ultrasonication with a probe-type sonicator (Bransonic B12) under nitrogen and cooling in ice-water (both positive and negative liposomes). Every $2 \mathrm{~min}$ sonication was interrupted for $1 \mathrm{~min}$ to let the dispersion cool down. Ultrasonication went on until no further decrease in turbidity was observed (usually after 10-20 min real ultrasonication time). Ultrasonication was followed by ultracentrifugation at $10^{\mathrm{s}} \mathrm{g}$ for $1 \mathrm{~h}$ at $4^{\circ} \mathrm{C}$. The supernatant containing small unilamellar vesicles (SUV) was used for further study. It was shown before (Crommelin et al., 1983) that for positive, doxorubicin-containing liposomes, filtration did not result in the expected size reduction. Therefore for positive liposomes this rechnique was excluded from the stability testing program. As a rule all liposome dispersions were kept protected from light at $4^{\circ} \mathrm{C}$.

\section{Determination of loading capacity}

For filtered, negatively charged MLV or SUV separation of free doxorubicin from liposome-associated doxorubicin was done by gel chromatography (Sephadex G50 fine). In positively and negatively charged multilamellar, non-filtered, liposome dispersions free doxorubicin was separated from bound doxorubicin by ultracentrifugation for $1 \mathrm{~h}$ at $10^{5} \mathrm{~g}$. For these dispersions gel chromatography was not possible as the liposomes tended to stick to the iop layer, clogging the column. For positive 
SUV an ircomplete recovery was found too. With this type of liposomes unsatisfactory gel chromatography results were reported earlier in the literature (Roerdink, 1978). To obtain doxorubicin binding data for positively charged SUV we therefore used a dialysis method. One $\mathrm{ml}$ of the dispersion was dialyzed for $24 \mathrm{~h}$ in a shaking bath under sink conditions in the receptor phase. A high ratio of exchange membrane area-to-volume of the dispersion was chosen $\left(3.2 \mathrm{~cm}^{2} / \mathrm{cm}^{3}\right)$. The dialysis membranes ( $M_{r} 10,000$ cut off; Diachema, Rüschlikon and Zürich) were soaked for $15 \mathrm{~min}$ in buffer and thereafter rinsed extensively before use. Twenty-four hours was sufficient for practically complete removal of free doxorubicin from the donor compartment. Doxorubicin leakage from the bilayer appeared to be negligible over this time span.

\section{Analytical methods}

After destruction of the liposomes by addition of Triton X-100, doxorubicin was assayed spectrophotometrically. Decomposition of doxorubicin taken up by liposomes on storage was determined by a HPLC method (Eksborg et al., 1979; modified by Oosterbaan et al., 1982). Phospholipid concentrations were determined by phosphate analysis according to Fiske and SubbarRow (1925). Particle size changes were monitored by turbidimetry $(700 \mathrm{~nm})$, or by determining the mean diameter by dynamic light scattering (Nanosizer, Coulter Electronics). Degrees of hydrolysis of the phospholipids were determined by TLC (Hauser, 1971). About 7 $\mu \mathrm{mol}$ of liposomes was chromatographed on precoated Kiezelgel $50, \mathrm{~F}_{254}$ (Merck) plates with a chloroform-methanol-water $(65: 35: 5)$ mixture. Phospholipids, lysocompounds and fatty acids were visualized by iodine vapour and scraped off the plate. Phospholipids and lyso-compounds were quantified by phosphate analysis after extraction of the compounds from the silica gel with methanol and evaporation of the extraction fluid. By this method it was found that egg phosphatidylcholine (PC) consisted for less than 2.5\% of lyso-compounds; for phosphatidylserine (PS) this percentage was below 5\%. For both positive liposomes after ultrasonication/ ultracentrifugation and negative liposomes after filtration through 600 and $200 \mathrm{~nm}$ pores these percentages were still below $3 \%$ after 14 days of storage.

Microelectrophoresis was carried out with a Mark 11 microelectrophoresis apparatus (Rank Bros.) as described before (Crommelin, submitted). క-Potentials were calculated from averaged mobilities of at least 30 liposomes in both directions and taken from both the upper and the lower stationary level.

\section{Results and Discussion}

\section{Optimization of doxorubicin loading capacity}

For positive and negative, non-filtered, MLV analogous curves representing the relationship between doxorubicin-liposome association and initia! ratio of the concentration of doxorubicin-to-phospholipid during film formaticn were found (Fig. 1A and B). For low ratios doxorubicin-liposome association grew with increasing ratio. Above certain ratios the curves levelled off and tended to plateau. 

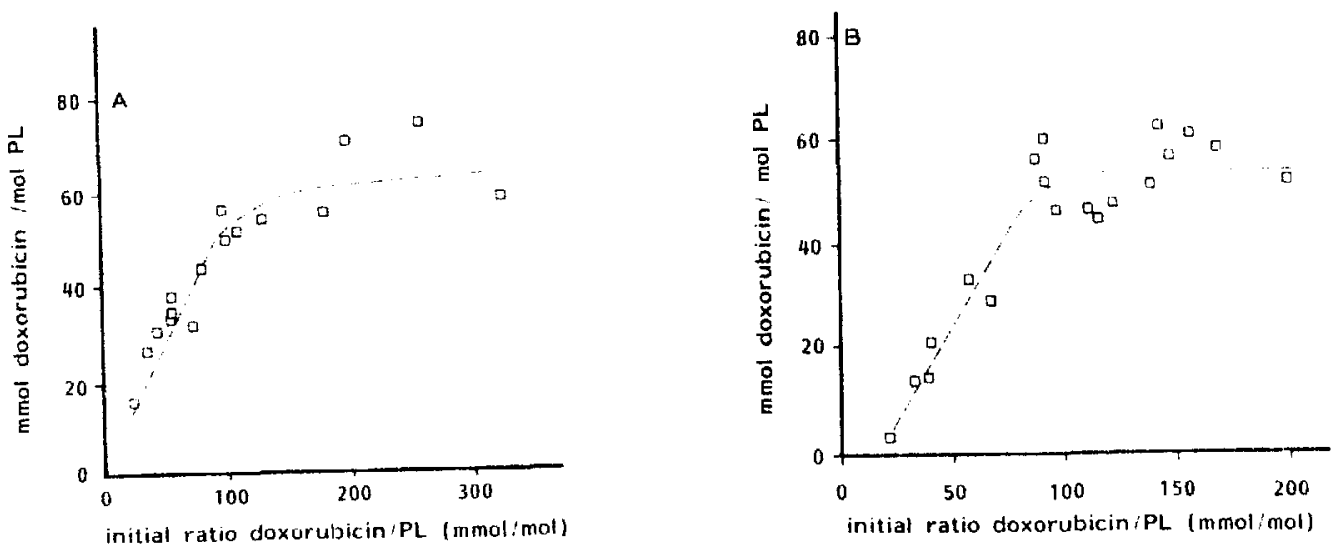

Fig. 1. A: loading efficiency ( $\mathrm{mmol}$ doxorubicin/mol PL) of negative liposomes as a function of the initial ratio of doxorubicin to $\mathrm{PL}$ ( $\mathrm{mmol} / \mathrm{mol}$ ). Loading efficiency of non-filtered, non-sonicated vesicles (MLV) cetermined 1 day after hydration of the film. Composition of the liposomes: PC-cholesterol-PS $(10: 4: 1)$. Concentration of PL varied between 3 and $30 \mathrm{mmol} / \mathrm{l} ; \mathrm{pH}$ of hydration medium: 4 . B: loading efficiency (mmol doxorubicin/mol PL) of positive liposomes as a function of the initial ratio of doxorubicin to $\mathrm{PL}(\mathrm{mmol} / \mathrm{mol})$. Loading efficiency of non-filtered, non-sonicated vesicles (MLV) determined one day after hydration of the film. Composition of the liposomes: $\mathrm{PC}$-cholesterol-stearylamine (10:4:3). Concentration of PL varied between 3 and $30 \mathrm{mmol} / 1 ; \mathrm{pH}$ of hydration medium: 4.

No more doxorubicin could associate with the bilayer; it was saturated. Encapsulation by the water phase played only a minor role in doxorubicin binding as can be illustrated with the followirg example. For negative liposomes with initial ratios of doxorubicin-to-phospholipid of 23 and $36 \mathrm{mmol}$ doxorubicin/mol PL and PL concentrations of $25 \mathrm{mmol} \mathrm{PL} / 167 \%$ and $72 \%$, respectively, of the total doxorubicin available was taken up by the liposomes. Determination of the capture volume (1 aqueous medium $/ \mathrm{mol} P L$ ) of our liposomes with the carboxyfluorescein technique (Weinstein et al., 1977) indicated that under the conditions mentioned before about $6 \%$ of the aqueous phase was encapsulated. This agreed well with literature data provided by Olson et al. (1979), obtained for a comparable type of liposome. Variation of the PL concentration between 3 and $30 \mathrm{mmol} P L / 1$ for a constant doxorubicin-to-PL ratio $(130 \mathrm{mmol} / \mathrm{mol})$ did not significantly change the loading capacity indicating that in that range, association with the liposomes was only dependent on the ratio doxorubicin-to-PL and not on the absolute concentrations in the aqueous phase. A reduction of the loading capacities was found after filtration of the negatively charged MLV through 600 and $200 \mathrm{~nm}$ membrane filters. In the investigated iange from 20 up to $180 \mathrm{mmol}$ doxorubicin/mol PL this decrease was independent of the initial ratio and proportional to the loading capacity of the unfiltered MLV: the mean loading capacity left was $75 \%$ of the "unfiltered capacity" $(n=5)$, coefficient of variation $20 \%)$. Ultrasonication reduced the doxorubicin-liposome association even more; for the negatively charged liposomes about $40 \%$ of the inicial 'unfiltered loading capacity' remained $(n=2)$. For positively charged liposomes, comparable results were found. But here even more variation occurred in the experimental data. This might be ascribed to the one-day time lapse between 
ultrasonication and assessment of the loading capacity after complete dialysis (see Methods and Materials) for the positive liposomes. As during dialysis the ultrasonicated liposomes were unstable (see below), more variables played a role than with the negative liposomes which were stable and could be assayed directly by gel chromatography. The substantial losses of doxorubicin during liposome sizing procedures surpassed the expected losses resulting from a decrease in capture volume because of the particle size reductions. The exact mechanism of the loss of loading capacity was not further investigated yet.

Release of doxorubicin from liposomes and stability of doxorubicin-containing liposomes on long-term storage

We studied both the release of doxorubicin and the changes in particle size of doxorubicin-containing liposomes after filtration or sonication (negative liposomes) and only sonication (positive liposomes).

Fig. 2 shows the results of the drug release on storage. Filtered, negatively charged, MLV ! ist over a 75-day period only 10\%; negatively charged SUV lost more of the drug: in 20 days about $40 \%$. For positive liposomes the $100 \%$ value was set after 12 days of dialysis. To determine doxorubicin release from positive liposomes samples of the stored dispersions were redialyzed at regular time intervals.

The mean diameter of filtered negatively-charged liposomes with doxorubicin after filtration through 600 and $200 \mathrm{~nm}$ pores as determined by dynamic light scattering was $0.27 \mu \mathrm{m}$ with a standard deviation of $0.01 \mu \mathrm{m}(\mathrm{n}=11)$, which was equal to liposomes of the same composition, prepared without doxorubicin: 0.28

Fig. 2.

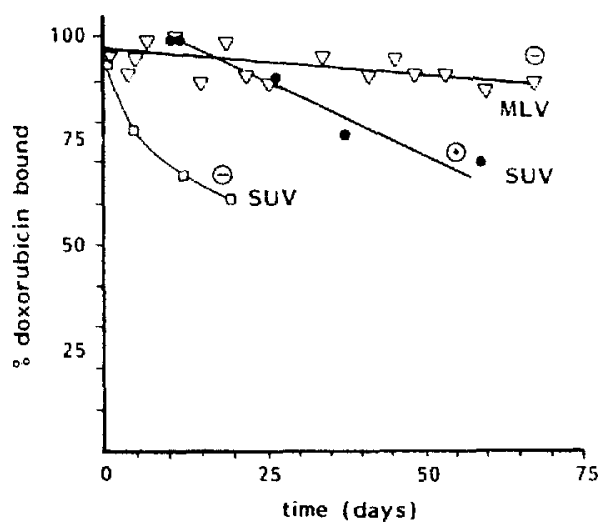

Fig. 3.

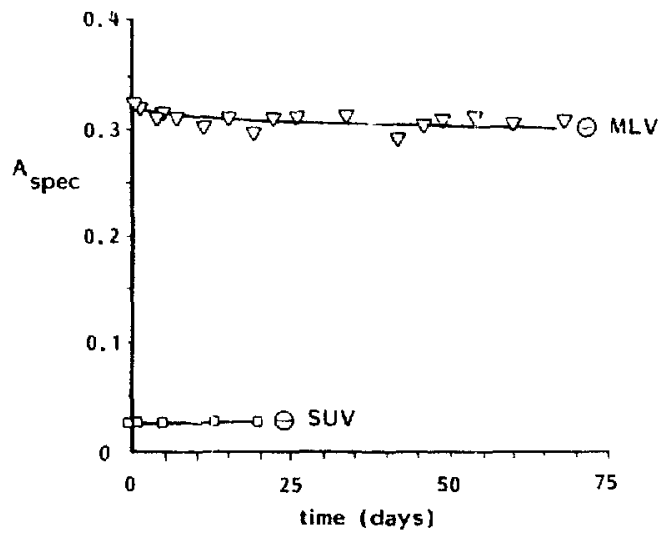

Fig. 2. Release of doxorubicin from liposomes on long-term storage. $\nabla$, filtered through 600 and $200 \mathrm{~nm}$ membrane filters, negative liposomes; $\square$, ultrasonicated and ultracentrifuged, negative liposomes; $\bullet$, ultrasonicated and ultracentrifuged, positive liposomes. Composition of negative liposomes: see Fig. 1A: initial doxorubicin/PL ratio: $80 \mathrm{mmol} / \mathrm{mol}$. Composition of positive liposomes: see Fig. 1B; initial doxorubicin/PL ratio: $140 \mathrm{mmol} / \mathrm{mol}$. Storage in refrigerator: $4-6^{\circ} \mathrm{C}$; mean of duplicate determinations.

Fig. 3. Specific absorbance of negative liposomes on long-term storage; for experimental conditions: see Fig. 2. $\nabla$, filtered through 600 and $200 \mathrm{~nm}$ membrane filters; $\square$, ultrasonicated and ultracentrifuged. 
$\mu \mathrm{m}$, standard deviation $0.01 \mu \mathrm{m}(\mathrm{n}=3)$. The specific turbidity $\left(\mathrm{A}_{\text {spec }}\right)$, defined as the absorbance at $700 \mathrm{~nm}$ per $\mu \mathrm{mol}$ PL per cm pathlength, was monitored over the same period of time as that used for following the release from the negatively charged liposomes (Fig. 3). No significant change was found. Dynamic light scattering data obtained at the start and at the end of the period of storage confirmed these turbidity results: a constant mean diameter of $0.27 \mu \mathrm{m}$ for the filtered, and $0.12 \mu \mathrm{m}$ for the ultrasonicated/ultracentrifuged dispersions. However, ultrasonicated and ultracentrifuged positive SUV were not stable. The $A_{\text {spec }}$ increased during the 12 days of dialysis from 0.04 to 0111 . Dynamic light scattering data indicated not only a growing mean particle diameter, but also an increasing heterodispersity as indicated by the polydispersity factor. Because of this instability the mean diameter for positive liposome dispersions (measured about 14 days after sonication and ultracentrifugation) showed much more variation than for the negative liposomes: 0.12 $\mu \mathrm{m}$. standard deviation $0.04 \mu \mathrm{m}(\mathrm{n}=6)$. Positive liposomes without doxorubicin tended to behave similarly: a poor reproducibility and a large heterodispersity.

We observed that one day after hydration positive (non-filtered) MLV containing doxorubicin consistently had mean diameters between 0.5 and $0.6 \mu \mathrm{m}$. This is different from positive (non-filtered) MLV without doxorubicin and negative (nonfiltered) MLV with or without doxorubicin, which had mean diameters of over $1 \mu \mathrm{m}$. Apparently doxorubicin affected the characteristics of the positively charged bilayer. Doxorubicin associated with negative liposomes showed little chemical decomposition over a period of 70 days of storage as measured by HPLC. After building the drug into positive liposomes, however, only a small fraction could be recovered after 30 days.

\section{Mechanisms of interaction}

The mechanism of interaction between doxorubicin and PL bilayers was investigated by microelectrophoresis. If doxorubicin, which is positively charged below $\mathrm{pH}$ 8 (Sturgeon and Schulman, 1977), interacted in ionized state with the bilayer, the negative potential of the negatively charged liposomes was expected to drop. Besides, interaction with positive liposomes was unlikely, because of mutual repulsion of the bilayer and drug ion. Therefore positive liposomes should contain not more doxorubicin than can be encapsulated in the aqueous phase. Fig. 4 shows the results of $\zeta$-potential measurements for positive and negative liposomes prepared with or without doxorubicin for different $\mathrm{pH}$ values.

For negative liposomes without doxorubicin the negative potential tended to increase slightly from $\mathrm{pH} 4$ to 7.4. This in contrast to the findings of Bangham et al. (1974), who reported no $\mathrm{pH}$-dependency of the negative charge in the range between $\mathrm{pH} 4$ and 7.4 for $10 \%$ PS containing liposomes without cholesterol. Schlieper et al. (1981) also found no significant $\mathrm{pH}$-dependency for PS vesicles between $\mathrm{pH} 4$ and $\mathrm{pH}$ 7.4. As doxorubicin was built into or adhered to the bilayer to a percentage of about $7 \%$ (on molecular basis) of the phospholipid content, an electrostatic binding mechanism was unlikely as virtually no difference in $\zeta$-potential was found for negative liposomes with or without doxorubicin. The $\zeta$-potential found for negative liposomes without doxorubicin complied well with the predicted $\%$-potential $(-12$ 


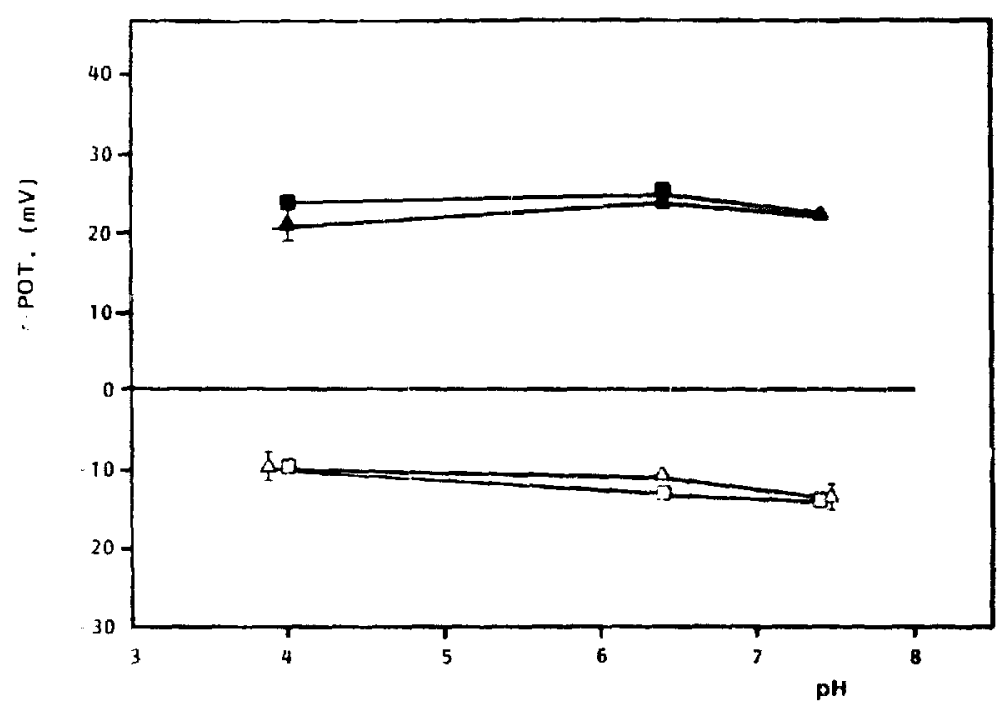

Fig. 4. $\zeta$-Potential of liposomes with or without doxorubicin as a function of the pH of the hylration medium. $\square$, "smpty". negative liposomes; $\Delta$, with doxorubicin, negative liposomes: $D$. "empty". positive" liposomes; $\Delta$, with doxorubicin, positive liposomes. For composition of the negative or positive liposomes: see Fig. 1A and B; initial doxorubicin/PL ratio for positive and negative liposomes 200 $\mathrm{mmol} / \mathrm{mol}$. To avoid an overcrowded microscopic view the dispersions were diluted with $0.01 \mathrm{M}$ Tris-HCl and $0.8 \%$ sodium chloride solutions with corresponding $\mathrm{pH}$. Verticle bars indicate the standard deviations $(n=3)$; otherwise the mean of duplicate determinations is given. For all individual suspensions the coefficients of variation of the velocities measured at the two stationary levels for both dircelions ucre less than 10\%. At each stationary level at least 30 observations of liposome velecities were made in buth directions. Experimental temperature: $25^{\circ} \mathrm{C}$.

$\mathrm{mV}$ ) assuming all PS molecules to be dissociated and taking into account a potential reduction because of sodium ion interaction ( $\mathrm{Nir}$ et al., 1978: Crommelin. submitted). For positive liposomes with doxorubicin, no influence of the $\mathrm{pH}$ of the aqueous medium on the $\zeta$-potential was found. Only for the $\mathrm{pH} 4$ and 6.3 cases we compared the $\zeta$-potentials in the presence of doxorubicin with those in the absence of the drug. No significant difference could be observed suggesting that doxorubicin was taken up into the bilayer mainly in the unionized form. The fact that doxorubicin (positively charged) associated with the positively charged bilayer for up to 5 t (mol. fraction) of the PL content, was additional support for a non-electrostatic interaction. In the literature conflicting results have been reported on the interaction between doxorubicin and phospholipid bilayers. Goldman et al. (1978) presented data on the interaction of doxorubicin and several analogs with phospholipid bilayers. They found both for DPPC- (dipalmitoylphosphatidylcholine) and PS-liposomes a strong interaction with doxorubicin. MLV consisting of DPPC or PS had a lower melting enthalpy in the presence of doxorubicin. No relation was found between the net charge of the compounds and the interaction with PS. From fluorescence spectra the authors deduced that doxorubicin was positioned at the water-hydrocarbon interface in SUV composed of PC and dicetylphosphate (9:1). On the other hand, Goormaghtigh et al. (1980a and b) reported a strong electrostatic interaction between doxorubicin and negatively charged phospholipids (cardiolipin and PS) in monolayer studies. No association was detected with neutral phospholipids. 
TABLE I

DOXORUBICIN-LIPOSOME INTERACTION IN CASE OF ADDITION OF DOXORUBICIN-HCI DISSOLVED IN THE HYDRATION MEDIUM TO THE PL-FILM

\begin{tabular}{|c|c|c|c|c|c|}
\hline \multirow[t]{2}{*}{ Charge } & \multirow{2}{*}{$\begin{array}{l}\text { Ratio of doxoru- } \\
\text { rubicin-to-PL } \\
\text { (mmol/mol) }\end{array}$} & \multicolumn{2}{|c|}{$\begin{array}{l}\text { Association of doxorubicin } \\
(\mathrm{mmol}) / \mathrm{PL}(\mathrm{mol})^{*}\end{array}$} & \multirow[t]{2}{*}{$\begin{array}{l}\text { doxorubicin } \\
\text { associated }\end{array}$} & \multirow[t]{2}{*}{$\begin{array}{l}\text { Encapsulated } \\
\text { water }^{\text {e }}\end{array}$} \\
\hline & & (b) & (c) & & \\
\hline+ & 170 & 20 & $\approx 50$ & 12 & 3 \\
\hline- & 170 & 61 & $\approx 60$ & 30 & 3 \\
\hline
\end{tabular}

Values presented are means of duplicate experiments. $\mathrm{pH}$ of hydration medium in 4.0

a $M V$, non-filtered, $24 \mathrm{~h}$ after hydration of the PL-film.

${ }^{n}$ Doxorubicin- $\mathrm{HCl}$ added in the hydration buffer to the PL-film.

- Doxorubicin- $\mathrm{HCl}$ partly dissolved in the methanol-chloroform mixture before film formation.

$\checkmark$ of total amount of doxorubicin present.

estimation based on capture volume data of liposomes of the composition used in this study without doxorubicin (oblained with carboxyfluorescein technique; Weinstein et al., 1977).

We investigated whether the stage of addition of doxorubicin to the phospholipids during liposome preparation was critical for the occurrence of the doxorubicin-phospholipid interaction. It was hypothesized that doxorubicin- $\mathrm{HCl}$ (partly) dissolved in the methanol-chloroform mixture (before film-formation) might initially interact in neutral (ion-pair) form with phospholipids. Alternatively. doxorubicin, dissolved in the aqueous medium, was added to the liposome film. We prepared PL films without doxorubicin and hydrated them with a doxorubicin containing Tris-sodium chloride solution of $\mathrm{pH} 4$. Here the drug is considered to be completely ionized. The initial ratio of doxorubicin-to-PL was chosen in the plateau zone of the curves in Fig. $1 \mathrm{~A}$ and B. The experimental data were compared with the expected doxorubicin association read from Fig. $1 \mathrm{~A}$ and $\mathrm{B}$.

Results are given in Table 1. For negative liposomes adding doxorubicin in the aqueous phase or in an earlier stage of preparation, i.e. the organic phase, to the phospholipids gave similar association data. Also after filtration or ultrasonication the loss of doxorubicin when added in the hydration medium was comparable to the situation reported above (not shown). For positive liposomes, however, doxorubicin added in the hydration medium of the phospholipid film interacted far less than when it was mixed with the phospholipids in the organic phase. Ultrasonication reduced the association even more; it approached the level obtained for hydrophilic compounds without any interaction with the bilayer.

Appirently in the case of positive liposomes doxorubicin is taken up much more effectively by bilayers when added in the organic phase where it is supposed to be present largely in neutral form. During hydration the positive charge of the lipid bilayer repelled the ionized doxorubicin and therefore interfered with the bilayer-drug interaction. This might be the explanation for the conflicting results mentioned before. Indeed, Goldman et al. (1978) added doxorubicin to the phospholipids before film formation, while Goormaghtigh et al. (1980a and b) investigated doxorubicin interactions with preformed monolayers and liposomes.

In conclusion. the uptake of doxorubicin tended to a plateau value of 60-75 
mmol doxorubicin/mol PL for negatively charged and $55 \mathrm{mmol}$ doxorubicin/mol PL for positively charged liposomes. Exceeding the limiting ratio of doxorubicin-toPL did not result in a higher loading and was therefore useless. In general doxorubicin was firmly bound to the bilayer; loss of the drug from the bilayer on storage increased in the following order: negative, filtered MLV, positive SUV. negative SUV. The negative liposome dispersions were physically stable, positively charged liposomes were not. An increase in mean particle size with a concurrent growth of the heterodispersity was observed. The mechanism of drug-liposome association seems to be rather complicated. Doxorubicin-containing liposomes had a similar $\zeta$-potential as the equivalent "empty" liposomes. So electrostatic interaction seemed unlikely if doxorubicin was present in the organic phase during PL film formation. If the drug was added to the bilayer dissolved in the hydration medium, no difference in binding capacity was found for the negative liposomes; however. for positive liposomes association with doxorubicin dropped dramatically.

\section{References}

Bangham, A.D., Hill, M.W. and Miller, N.G.A.. Preparation and use of liposomes as models of biological membranes. In Korn, E. (Ed.), Methods in Membrane Biology. Vol. 1, 1974. pp. 1-68.

Crommelin, D.J.A. Slaats, N. and van Bloois, L. Preparation and characterization of doxorubicin containing liposomes: 1. Influence of liposome charge and $\mathrm{pH}$ of hudration medium on loading capacity and particle size. Int. J.-Pharm., 16 (1983) 79-92.

Crommelin, D.J.A., Influence of lipid composition and ionic strength on the physical stability of liposomes. J. Pharm. Sci., submitted.

Eksborg, S., Ehrscon, H. and Andersson, I. Reversed phase liquid chromatographic determination of plasma levels of Adriamycin and Adriamycinol. J. Chromatogr., 16.1 (1979) 479-486.

Fiske, C.H. and SubbarRow, Y., The colorimetric determination of phosphorus. J. Biol. Chem.. 66 (1925) 375 .

Forssen. E.A. and Tökes, Z.A., Use of anionic liposomes for the reduction of chronic doxorubicin-induced cardiotoxicity. Proc. Natl. Acad. Sci. U.S.A., 78 (1981) 1873-1877.

Gabizon, A., Dagan, A., Goren, D., Bahrenholz, Y. and Fuks, Z. Liposomes as in vivo carriers of adriamycin: reduced cardiac uptake and preserved antitumor activity in mice. Cancer Res.. 42 (1982) 4734-4739.

Goldman, R., Facchinetti, T., Bach, D., Raz, A.. and Shinitzky. M.. A differential interaction of daunomycin, adriamvcin and their derivatives with human erythrocytes and phospholipid bilavers. Biochim. Biophys. Acta, 512 (1978) 254-269.

Goormaghtigh, E., Chatelain, P., Caspers, J. and Ruysschaert, J.M.. Evidence of a complex between adriamycin derivatives an. ${ }^{1}$ cardiolipin: possible role in cardiotoxicity. Biochem. Pharmacol. 29 (1980) 3003-3010.

Goormaghtigh, E., Chatelain, P., Caspers, J. and Ruysschaert. J.M., Evidence of specific complex between adriamycin and negatively charged phospholipids. Biochim. Biophys. Acta. 507 (1980) 1-14.

Hauser. H.O. The effect of ultrasonic irradiation on the chemical siructure of egg lecithin. Biochem Biophys. Res. Commun., 45 (1971) 1049-1055.

Nir, S. and Bentz, J., On the forces between phospholipid hilayers. J. Colloid Interface Sci.. 65 (1978) 399-414.

Olson. F., Hunt, C.A., Szoka, F., Vail, W.J. and Papahadjopoulos. D., Preparation of liposomes of defined size distribution by extrusion through polycarbonate membranes. Biochim. Biophys. Acta. 557 (1979) 9-23.

Olson. F.. Mayhew, E., Maslow, D., Rusium, Y. and Szoka, F., Characterization. toxicity and therapeutic efficacy of adriamycin encapsulated in liposomes. Eur. J. Cancer Clin. Oncol. 18 (1982) 167-176. 
Oosterbaan, M.J.M., Dirks, M.J.M., Vree, T.B., van der Kleyn, E., Simonetti, G.S. and McVie, J.G., Klinische farmacokinetiek van adriamycine. Tijdschr. Geneesmiddelenonderzoek, 7 (1982) 1372-1378.

Rahman, A., Kessler. A., More, N., Sikic, B., Rowden, G., Woolley, P. and Schein, P.S., Liposomal protection of adriamycin-induced cardiotoxicity in mice. Cancer Res., 40 (1980) 1532-1537.

Roerdink, F.H., Liposomes as enzyme carriers, Thesis, Groningen, 1978.

Schlieper, P., Medda, P.K. and Kaufmann, R., Drug-induced zeta-potential changes in liposomes studied by laser doppler spectroscopy. Biochim. Biophys. Acta, 644 (1981) 273-283.

Sturgeon, R.J. and Schulman, S.G., Electronic absorption spectra and protolytic equilibria of doxorubicin: direct spectrophotometric determination of micraconstants. J. Pharm. Sci., 66 (1977) 958-961.

Weinstein, J.N., Yoshikami, S., Henkart, P.A., Blumenthal, R. and Hagins, W.A., Liposome-cell interaction: transfer and intracellular release of a trapped fluorescent marker. Science, 195 (1977) 489-491. 